\title{
Correction to: Breastfeeding Practices of Ethnic Minorities in China: A Population-Based Cross-Sectional Study of 10,408 Mothers
}

\author{
Yu Zhang ${ }^{1,3} \cdot$ Hanyu Wang ${ }^{1} \cdot$ Yiqing Wang ${ }^{2} \cdot$ Kun Tang $^{3}(\mathbb{D}$
}

Published online: 13 August 2019

(C) The Author(s) 2019

\section{Correction to: Journal of Immigrant and Minority Health https://doi.org/10.1007/s10903-019-00918-1}

The article Breastfeeding Practices of Ethnic Minorities in China: A Population-Based Cross-Sectional Study of 10,408 Mothers, written by Yu Zhang, Hanyu Wang, Yiqing Wang and Kun Tang, was originally published electronically on the publisher's internet portal (currently SpringerLink) on July 19, 2019 without open access.

With the author(s)' decision to opt for Open Choice the copyright of the article changed on August 12, 2019 to $($ ) The Author(s) 2019 and the article is forthwith distributed under the terms of the Creative Commons Attribution 4.0 International License (http://creativecommons.org/licenses/ by/4.0/), which permits use, duplication, adaptation, distribution and reproduction in any medium or format, as long as you give appropriate credit to the original author(s) and

Yu Zhang and Hanyu Wang are co-first authors.

The original article can be found online at https://doi.org/10.1007/ s10903-019-00918-1.

Kun Tang

tangk@mail.tsinghua.edu.cn

Yu Zhang

yuzhang_@pku.edu.cn

Hanyu Wang

whybest@pku.edu.cn

Yiqing Wang

wangyiqing@bjmu.edu.cn

1 School of Health Humanities, Peking University Health Science Center, No. 38 Xueyuan Rd, Haidian District, Beijing 100084, People's Republic of China

2 School of Basic Medical Sciences, Peking University Health Science Center, Xueyuan Rd, Haidian District, No. 38, Beijing 100084, People's Republic of China

3 Research Center for Public Health, Tsinghua University, Haidian District, Beijing 100084, People's Republic of China the source, provide a link to the Creative Commons license and indicate if changes were made.

Publisher's Note Springer Nature remains neutral with regard to jurisdictional claims in published maps and institutional affiliations. 